\title{
Importance of Elastic Recovery in the DSR for Binders and Mastics
}

\author{
Cristian S. Clopotel ${ }^{\mathrm{a} *}$ and Hussain U. Bahia ${ }^{\mathrm{b}}$ \\ Department of Civil and Environmental Engineering, University of Wisconsin Madison, 1415 Engineering \\ Dr., Madison, Wisconsin 53706, USA \\ E-mail: clopotel@wisc.edua,*, bahia@engr.wisc.edu ${ }^{\mathrm{b}}$
}

\begin{abstract}
Elastic Recovery (ER) is used by many highway agencies for modified bitumen grading specifications. The current ASTM/AASHTO standards for ER testing utilize the ductility bath as the measurement apparatus. A new procedure for measuring the elastic recovery in the Dynamic Shear Rheometer (ER-DSR) was developed and was used to test different polymer modified binders and mastics. The results show a good correlation between the ER-DSR and ER measured in the ductility bath (ER-DB). The ER-DSR requires less material and is more time efficient and repeatable than the ER-DB. Since the link between the ER measurements and pavement performance is not well established, the study compared ER measurements with binder rutting and fatigue results as measured by the Multiple Stress Creep and Recovery (MSCR) test and the linear amplitude sweep (LAS) test, respectively. Generally, binders and mastics with higher ER-DSR value showed higher percent recovery (MSCR) and fatigue resistance (LAS). This study also showed the effect of natural and manufactured fillers on the percent recovery measured with the MSCR test. Based on the experimental results, it is recommended to replace ER-DB with the ER-DSR. Also, agencies should consider the MSCR and fatigue tests as indicators of binder modification rather than the ER, as they provide direct measurements of damage resistance characteristics for which the elastic recovery might not indicate.
\end{abstract}

Keywords: Elastic recovery, multiple stress creep and recovery (MSCR), polymer modified asphalt binder, mastics, fatigue.

ENGINEERING JOURNAL Volume 16 Issue 4

Received 4 January 2012

Accepted 2 April 2012

Published 1 July 2012

Online at http://www.engj.org/

DOI:10.4186/ej.2012.16.4.99 


\section{Introduction}

Current PG grading methods are not appropriate for measuring mechanical properties of modified binders beyond the linear viscoelastic range. The current Superpave protocols worked well for regular unmodified binders, however, have been shown to be inadequate for polymer modified binders. The National Cooperative Highway Research Program (NCHRP) Project 9-10, [1] "Superpave Protocols for Modified Asphalt Binders," was initiated to confirm whether the current Superpave protocols are suitable for use with modified asphalt binders. The conclusion drawn was that the Superpave specifications cannot be used to fully characterize all asphalt binders modified with different additives. The main reason is that they are based on simplifying assumptions that cannot be reliably extended to modified binders. Consequently, many state agencies introduced additional tests to characterize polymer modified binders, such as elastic recovery and forced ductility. These tests along with the Performance Grade (PG) specifications comprise what is called the Superpave Plus specifications[2, 3].

In 2006 at the Association of Modified Asphalt Producers, Casola [4] presented a survey regarding the modified asphalt market. According to this survey $81 \%$ of the state agencies implemented Superpave Plus specifications and $60 \%$ of these agencies require elastic recovery. The advocates of the elastic recovery test claimed that in addition to determining the presence of modifiers, this test can also provide information about the material quality. The testing procedure for the elastic recovery is standardized in the AASHTO T301 "Elastic Recovery Test of Bituminous Materials by Means of a Ductilometer" [5] and the ASTM D6084 "Standard test method for elastic recovery of bituminous materials by ductilometer" [6]. Although the test is standardized, many state agencies use alternative procedures to measure elastic recovery [7]. These changes can be attributed to the concerns about conducting the standard test such as time to run the test, also the relationship between binder elastic recovery and performance properties are not fully understood.

In this paper, a novel test procedure using the Dynamic Shear Rheometer (DSR) to measure elastic recovery of asphalt binders and mastics is proposed. Recovery results were related to rutting and fatigue performance obtained from the Multiple Stress Creep and Recovery (MSCR) test and the linear amplitude sweep (LAS) test, respectively.

\section{Objectives}

The main objectives of this study are to:

- Develop a simple protocol for measuring the elastic recovery in the DSR (ER-DSR),

- Compare the results of the new test with the elastic recovery measured in the ductility bath (ER$\mathrm{DB})$,

- Correlate the elastic recovery measurements with binder rutting resistance results obtained from the Multiple Stress Creep and Recovery (MSCR) test; and fatigue resistance results obtained from linear amplitude sweep.

- Determine the effect of mineral fillers of the elastic recovery of asphalt binders.

\section{Materials and Methods}

\subsection{Material Sources and Preparation}

Materials selected for this study included:

- Two different base binders, Flint Hills (FH) PG 64-22, and CRM PG 58-28.

- Seven modifier types: linear styrene-butadiene-styrene block copolymer (LSBS), radial styrenebutadiene-styrene block copolymer (RSBS), terpolymer-Elvaloy 4170, terpolymer-Elvaloy AM, two oxidized polyethylene generically called PE1 and PE2, and polyphosphoric acid(PPA).

- Three mineral fillers: limestone (LS2), dolomite (DS2) and caliches (CA2)

- Two levels of modification: one grade jump, and two grade jumps.

The selected modifiers cover a broad range of modification techniques: reactive and non-reactive elastomers, cross-linked and uncross-linked polymeric additives, plastomers, and a low molecular weight acid modifier. All the blends were performed in the lab following the modifiers manufacturer guidelines. The required amount of polymer to achieve the one and two grade jumps was determined based on trial 
and error. For this study, three asphalt binders were selected to be blended with the mineral fillers: FH neat, FH modified with 4\% RSBS and sulfur and FH modified with 1.5\%Elvaloy AM and PPA. All the mastics were produced by blending the mineral fillers with the RTFO aged asphalt binder. Based on the findings from the NCHRP project 9-45,"Test Methods and Specification Criteria for Mineral Filler Used in HMA" [8], a $28 \%$ volume fraction for the fillers was used to calculate the required amount of filler. Fillers with different Rigden voids were selected for this study: low (DS2), medium (LS2), high (CA2). The properties of the fillers are shown in Table 1.

Table 1. Filler properties.*

\begin{tabular}{lcccccccr}
\hline Code & $\begin{array}{c}\text { Rigden } \\
\text { Voids (\%) }\end{array}$ & $\begin{array}{c}\text { Fineness } \\
\text { Modulus }\end{array}$ & $\begin{array}{c}\text { CaO } \\
\mathbf{( \% )}\end{array}$ & $\begin{array}{c}\text { Methylene } \\
\text { Blue } \\
\text { Volume }\end{array}$ & $\begin{array}{c}\text { Specific } \\
\text { Gravity }\end{array}$ & $\begin{array}{c}\text { Lost On } \\
\text { Ignition } \\
\mathbf{( \% )}\end{array}$ & $\begin{array}{c}\text { Plasticity } \\
\text { Index }\end{array}$ & $\begin{array}{r}\text { Solubility } \\
(\%)\end{array}$ \\
\hline DS2 & 29.4 & 4.73 & 27.00 & 1.82 & 2.70 & 0.35 & Non-Plastic & 0.7 \\
LS2 & 35.4 & 3.68 & 46.30 & 3.87 & 2.62 & 0.04 & Non-Plastic & 1.5 \\
CA2 & 45.0 & 5.13 & 40.00 & 10.25 & 2.49 & 1.35 & Non-Plastic & 1.0 \\
\hline
\end{tabular}

*From NCHRP 9-45[8].

\subsection{Test Methods and Procedures}

\subsubsection{Elastic Recovery in the DSR}

The protocol used for running the elastic recovery in the DSR for asphalt binder and mastics can be summarized as follows:

- RTFO aged binder sample is heated in the oven until is fluid enough to be poured into a silicon mold. The procedure follows the ASTM D7175 "Standard Test Method for Determining the Rheological Properties of Asphalt Binder Using a Dynamic Shear Rheometer" [9].

- The binder sample is transferred from the silicon mold to the DSR plate. The gap is set to $2.10 \mathrm{~mm}$ to allow for trimming the excess binder from the sample. The gap is then set to $2 \mathrm{~mm}$.

- The temperature of the sample is kept at the required temperature $\left(25^{\circ} \mathrm{C}\right)$ for 20 minutes. This step is required to reach thermal equilibrium.

- A constant strain rate of $0.0231 / \mathrm{s}$ is applied for 2 minutes. This step is run in strain controlled mode.

- A constant zero shear stress is applied for a period of 1 hour. This step is run in stress controlled mode and corresponds to the relaxation part of the test.

A typical plot for the shear strain versus time in the DSR is presented in Fig. 1. The elastic recovery at the end of the relaxation step is calculated as follows:

$$
\text { ER-DSR }=\frac{\text { recovered strain at the end of relaxation } \operatorname{step}\left(\varepsilon_{2}\right)}{\text { strain at the end of loading } \operatorname{step}\left(\varepsilon_{1}\right)} \times 100 \%
$$




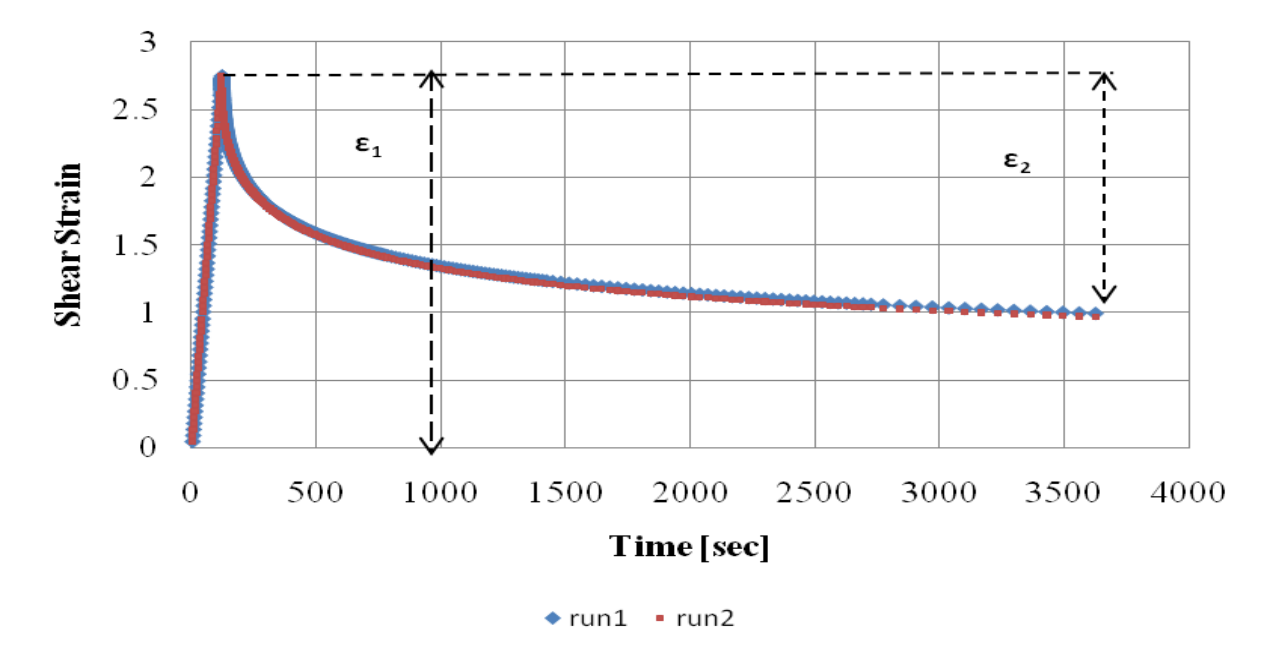

Fig. 1. Typical shear strain curve for the elastic recovery test in the DSR.

\subsubsection{Linear Amplitude Strain (LAS)}

The LAS test is used to evaluate the ability of asphalt binder to resist fatigue damage by applying cyclic loading at increased amplitudes. The LAS test protocol consists of two steps: in the first step the undamaged rheological properties are measured while the damage characteristics of the binder are measured in the second step.

The undamaged property of the binder is represented by the "alpha" parameter $(\alpha)$ and it is indirectly determined from a frequency test. The $\alpha$ parameter is calculated as $1 /(1+\mathrm{m})$, where $\mathrm{m}$ is the maximum slope of the relaxation modulus $G(t)$ versus time plot in logarithmic scale $[10,11,12]$. The second step of the protocol employs an amplitude sweep test. The test is run in a strain-controlled mode using oscillatory shear, at a frequency of $10 \mathrm{~Hz}$. The loading scheme consists of 10 second intervals of constant strain amplitude followed by another interval of increased strain amplitude. The strain amplitude is increased from $0.1 \%$ to $20 \%$. Peak shear strain and peak shear stress is recorded every 10 load cycles, along with phase angle and dynamic shear modulus [10]. The fatigue life is calculated using the following equation:

$$
\mathrm{N}_{\mathrm{f}}=\mathrm{A}\left(\gamma_{\max }\right)^{\mathrm{B}}
$$

where $\mathrm{A}$ and $\mathrm{B}$ are coefficients that depend on material characteristics which were calculated using viscoelastic continuum damage theory (VECD). The test was performed at the intermediate temperatures determined according to the AASHTO MP1" Standard Specification for Performance Graded Asphalt Binder" [13]. More details about LAS test can be found in [10].

\subsubsection{Multiple Stress Creep and Recovery (MSCR) Test}

The MSCR test was run according to the ASTM 7045-10 "Standard Test Method for Multiple Stress Creep and Recovery (MSCR) of Asphalt Binders using a Dynamic Shear Rheometer" [14]. According to this standard the binder sample is loaded at a constant creep stress for 1 second followed by a zero stress recovery of 9 seconds. Ten cycles of creep and recovery are run at $0.1 \mathrm{kPa}$ creep stress followed by ten at $3.2 \mathrm{kPa}$ creep stress. The non-recoverable compliance Jnr and percent recovery after ten cycles at $0.1 \mathrm{kPa}$ and $3.2 \mathrm{kPa}$ are reported. The testing temperature was selected according to the AASHTO MP19 "Performance-Graded Asphalt Binder Using Multiple Stress Creep Recovery (MSCR) Test" [15], and was $64^{\circ} \mathrm{C}$ for the FH binders and $58^{\circ} \mathrm{C}$ for the CRM binders. All testing was performed on RTFO aged binders.

\section{Results and Discussion}

\subsection{Correlation between Elastic Recovery in the Ductility Bath and Elastic Recovery in the DSR}

The relation between elastic recovery in the ductility bath and elastic recovery in the DSR for asphalt binder and mastics are shown in Fig. 2 and Fig. 3, respectively. As shown very good correlations between these 
tests were observed for both binder and mastics $\left(\mathrm{R}^{2}=0.97\right.$ and $\mathrm{R}^{2}=0.96$, respectively). The results also showed that the mineral fillers reduce elastic recovery.

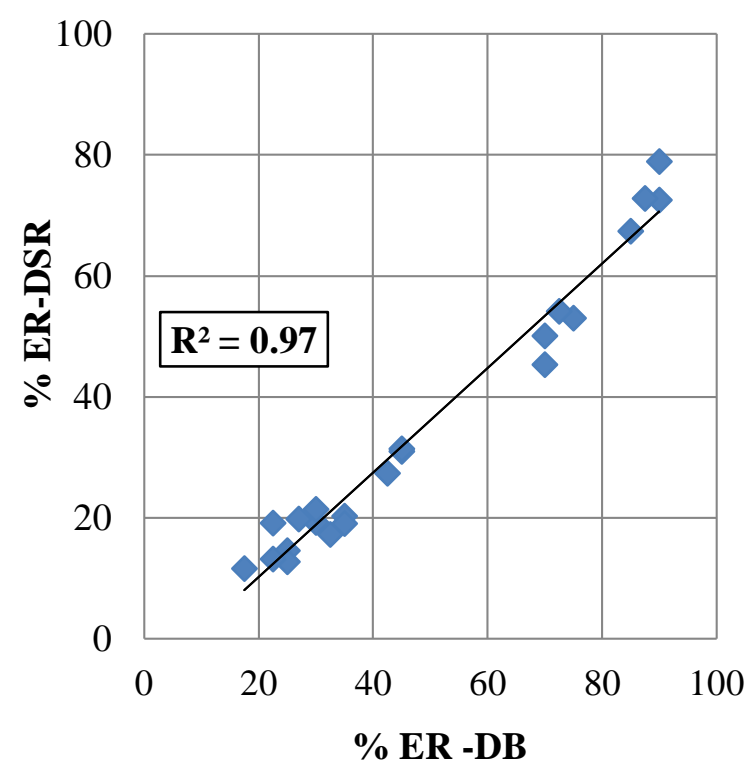

Fig. 2. Correlation between ER-DSR and ER-DB for asphalt binder.

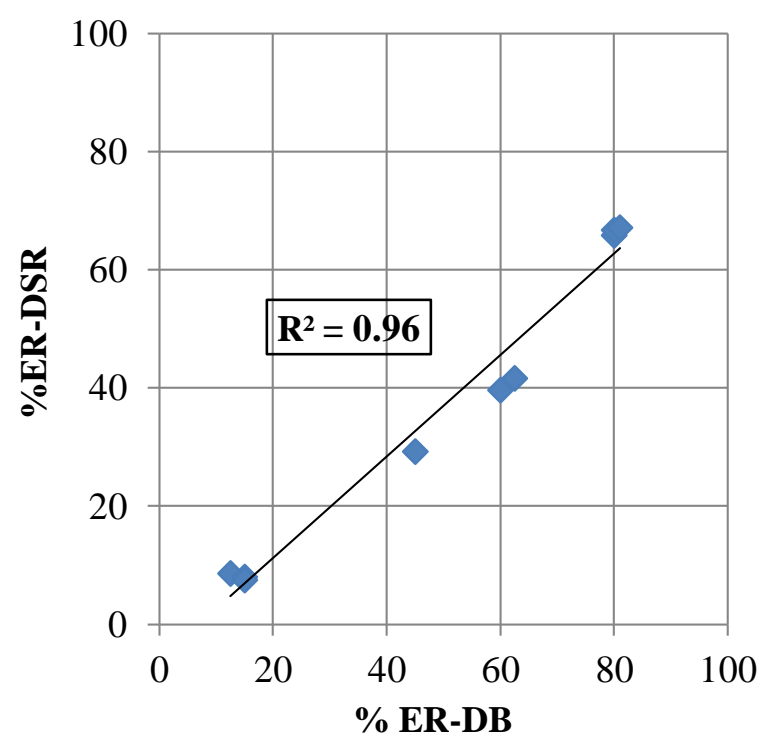

Fig. 3. Correlation between ER-DSR and ER-DB for mastics.

The testing results for the binders and mastics showed that the ER-DSR is on average $70 \%$ lower than ER-DB. The reasons for this shift can be attributed to the following factors: mode of loading, strain rate, sample geometry, temperature control, and operator sensitivity. The load in the DSR is applied in shear while the load in ductility bath is applied in tension. The DSR allows better control of the testing temperature than the ductility bath. The temperature in the DSR was maintained within a $\pm 0.1^{\circ} \mathrm{C}$ range. On the other hand the elastic recovery in the ductility uses an open bath, and the temperature is highly dependent on the environmental conditions. However, the tests were performed according to the current standard which requires the temperature to be controlled within $\pm 0.5^{\circ} \mathrm{C}$. Finally, measurements in the DSR are completely automated while the measurements in the ductility bath are highly operator dependent.

One of the biggest advantages of running the elastic recovery in the DSR is the possibility of continuous monitoring of the results. The elastic recovery procedure collects data every 0.065 seconds, and thus it is possible to determine the elastic recovery at different time intervals: 15, 30, 45 and 60 minutes. Good correlations were also obtained between ER-DB and ER-DSR measured at 15, 30, 45 minutes of relaxation time. An ANOVA analysis showed that ER-DSR measured at 15 minutes is significantly different from ER-DSR at 60 minute ( $\mathrm{p}$-value $=0.01$ ), while the difference between the ER-DSR at 30 minutes and ER-DSR at 60 minutes is not significant $(p-v a l u e=0.64)$. Based on these results it is recommended that the relaxation part can be reduced to 30 minutes.

\subsection{Effect of Mineral Filler on Elastic Recovery Properties}

Three mineral fillers were selected for this study based on Rigden voids values. The ER-DSR results for different mineral fillers are shown in Fig. 4. The results pointed out that for the unmodified and RSBS modified binder, the elastic recovery was very similar regardless of the filler type used. No significant differences in the elastic recovery were observed for the FH $+1.5 \%$ Elvaloy $\mathrm{AM}+\mathrm{DS} 2$ and $\mathrm{FH}$ $+1.5 \%$ Elvaloy AM + LS2. The elastic recovery for FH $+1.5 \%$ Elvaloy AM + CA2 is on average $30 \%$ lower than for the FH $+1.5 \%$ Elvaloy AM + DS2 and FH +1.5\%Elvaloy AM + LS2. 


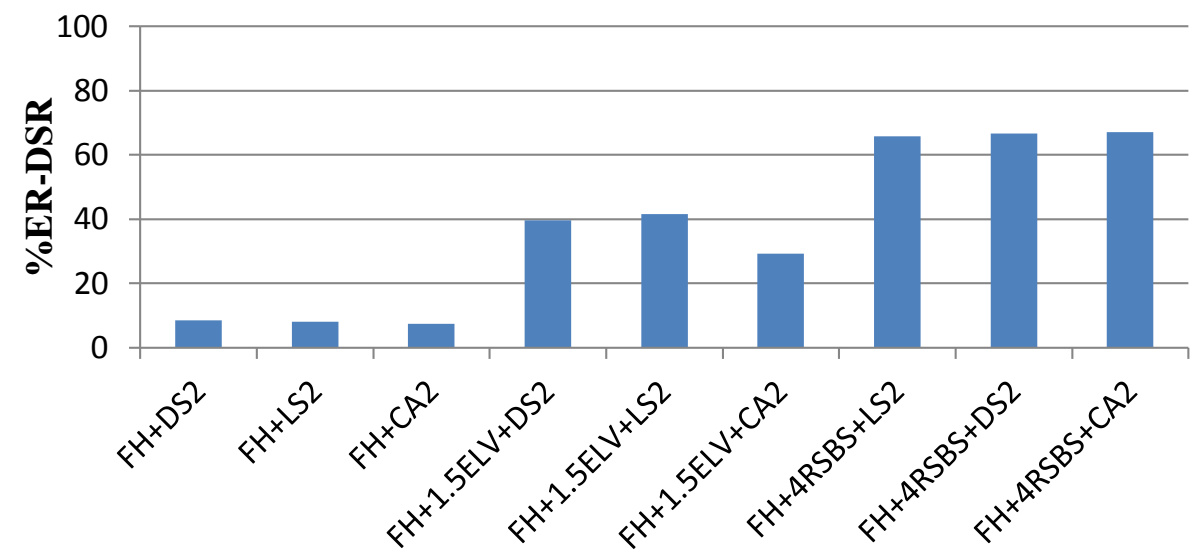

Fig. 4. Effect of the mineral filler on elastic recovery.

\subsection{Relationship between ER-DSR and Fatigue \& Rutting Binder Performance}

Although elastic recovery test is widely used, there is significant debate about the real value of this test and why is being used. Some experts claim that the best benefit is assuring users of modified binders that polymers are used; others claim that elasticity is an indication of durability and general quality. There is no doubt that this test is a good indicator of the use of elastomeric polymers, but it is not a good indicator of other types of polymers and it is difficult to find direct evidence that elastic behavior is related to durability or general quality. It is also difficult to find evidence that elasticity is an indicator of poor quality. In this study, a comparison between the ER-DSR and test results from MSCR (rutting) and LAS (fatigue) was obtained, to determine the value of the ER as a performance indicator.

\subsection{Relation between the Elastic Recovery in the DSR and MSCR Results}

Figure 5(a) and Figure 5(b) show the correlation between the ER- DSR at $25^{\circ} \mathrm{C}$ and percent recovery from MSCR test (\%R) at $3.2 \mathrm{kPa}$ for asphalt binder and mastics, respectively. As shown, good correlation was obtained for both asphalt binder and mastics. Additional testing might be required in order to validate the relationship between $\% \mathrm{R}$ and $\%$ ER-DSR for mastics.

\subsection{Relation between the Elastic Recovery in the DSR and Linear Amplitude Strain Results}

Recently the Linear Amplitude Sweep test has been proposed as an effective surrogate for measuring binder fatigue resistance and as a possible binder fatigue specification [10]. The relation between elastic recovery and the number of cycles to failure at 2.5\% strain (i.e., $\mathrm{Nf} @ 2.5 \%$ ) for the asphalt binder and mastic is shown in Fig. 6(a) and Fig. 6(b), respectively. It can be seen that there is a good trend indicating that higher ER values are better for fatigue. There is however a relatively large scatter that makes the substitution of $\mathrm{ER}$ values for the $\mathrm{Nf}$ values very questionable. 


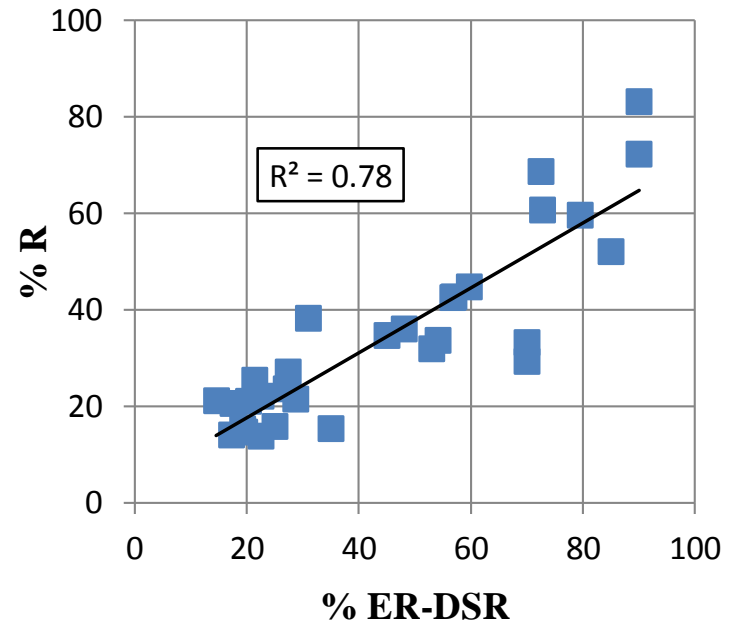

(a) asphalt binder

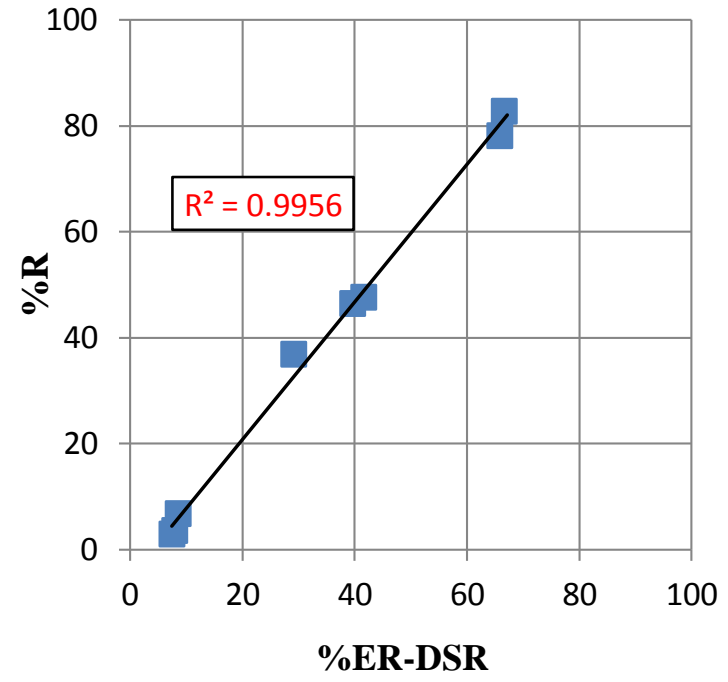

(b) mastics

Fig. 5. Correlation between ER-DSR and percent recovery from MSCR at $3.2 \mathrm{kPa}$.

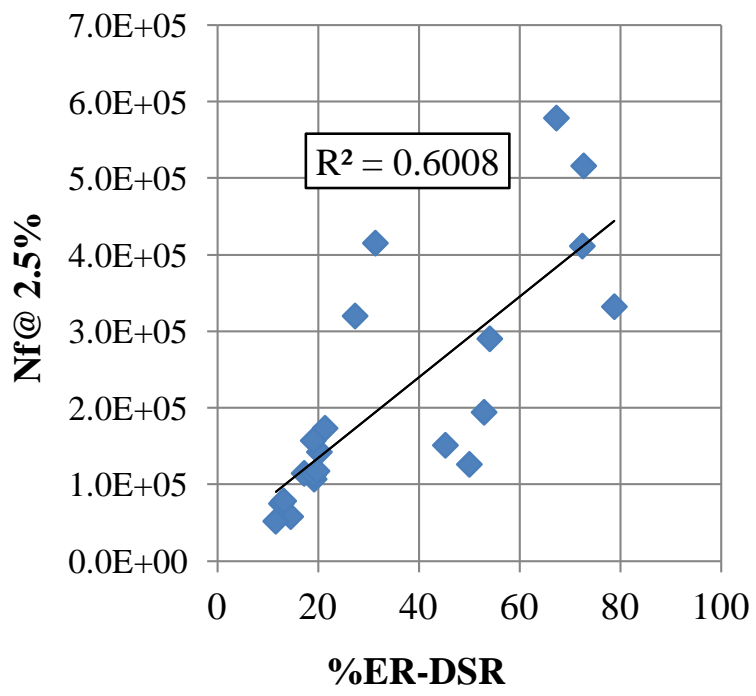

Fig. 6(a). ER-DSR vs. Nf@2.5\% -asphalt binder.

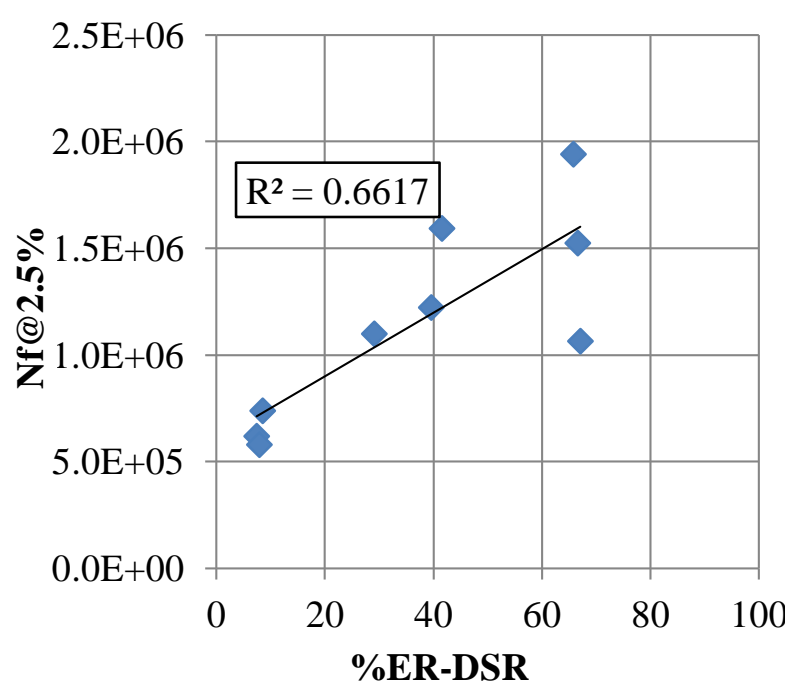

Fig. 6(b). ER-DSR vs. Nf@2.5\%-mastics.

\section{Conclusions}

The elastic recovery (ER) measurements have been used to compliment the PG grading by many transportation agencies. There have been many concerns regarding the details of the current standards AASHTO T301/ASTM D 6084 procedures. In this study, a new procedure for measuring elastic recovery in the DSR was developed and used to measure the response for polymer modified binders and mastics. The DSR-ER results were first correlated to the standard ductility bath test for elastic recovery. To assess the value of the elastic recovery in relating to performance, ER values measured with the DSR were compared with the fatigue results collected from linear amplitude strain sweep test as well as the MSCR results. Based on the results the following conclusions could be drawn:

- The elastic recovery measured in the ductility bath can be replaced by elastic recovery measured in the DSR. A very good correlation between these tests was obtained for both asphalt binder and mastics $(\mathrm{R} 2=0.97$ and $\mathrm{R} 2=0.96$ respectively).

- The correlation obtained between the ER-DSR and ER-DB remained very good also after 30 minute relaxation time. It is recommended to use a 30 minutes relaxation time. 
- The correlations between ER-DSR and number of cycles to failure from the linear amplitude sweep (LAS) results, were relatively poor and thus using ER as a surrogate test for fatigue characterization does not appear to be promising.

- The correlation between ER-DSR and \%recovery from the MSCR test at $3.2 \mathrm{kPa}$ for both asphalt binder and mastics was good. Higher variability is observed for the binder results in comparison to mastic testing.

In summary, the study leads to recommend that the procedure for measuring the elastic recovery in the ductility bath be replaced with the DSR. The new procedure has the following advantages: small sample size, reduction in the testing time, easy preparation and automated measurements. The results indicate that some correlation exists between ER-DSR and certain binder fatigue properties.

\section{References}

[1] H. U. Bahia, D. I. Hanson, M. Zeng, H. Zhai, M. A. Khatri, and M. R. Anderson, "Superpave protocols for modified asphalt binders," Prepared for the National Cooperative Highway Research Program Transportation Research Board, National Research Council, Project NCHRP 9-10, Final Rep., 2000

[2] J. D'Angelo and R. Dongre, "Superpave binder specifications and their performance relationships to modified binders," in Proceedings Canadian Technical Asphalt Association, 2002, vol. 47, pp. 91-104

[3] N. I. Kamel, H. U. Bahia, and D. W. Cho, "Critical laboratory evaluation of asphalt binders modified by refining processes," in Proceedings of Canadian Technical Asphalt Association, 2004, pp. 57-76.

[4] J. Casola. (2006). Modified Alphalt Market Survey. [Online]. Available: http://www.modifiedasphalt.org/papers/Casola06.pdf

[5] Elastic Recovery Test of Bituminous Materials by Means of a Ductilometer, AASHTO T301, 2008.

[6] Standard Test Method for Elastic Recovery of Bituminous Materials by Ductilometer, ASTM Standard D6084, 2006.

[7] J. A. D’Angelo, R. Dongre, and G. Reinke, "Creep and recovery," Public Roads, vol. 70, no. 5, pp. 2430, 2007.

[8] H. U. Bahia and A. Faheem, "Test methods and specification criteria for mineral filler used in HMA," Project NCHRP 9-45, Draft Rep., 2010.

[9] Standard Test Method for Determining the Rheological Properties of Asphalt Binder Using a Dynamic Shear Rheometer, ASTM Standard D7175, 2008

[10] C. Johnson, "Evaluation of accelerated procedures for fatigue characterization of asphalt binders," Ph.D. Thesis, University of Wisconsin, Madison, 2010.

[11] H. J. Lee, "Viscoelastic constitutive model for asphalt concrete under cyclic loading," Journal of Engineering Mechanics, vol. 124, no. 32, 1998.

[12] S. W. Park, Y. R. Kim, and R. A. Schapery, "A viscoelastic continuum damage model and its application to uniaxial behavior of asphalt concrete," Mechanics of Materials, vol. 24, no. 4, pp. 241-255, 1996.

[13] Standard Specification for Performance Graded Asphalt Binder, AASHTO MP1, 1998.

[14] Standard Test Method for Multiple Stress Creep and Recovery (MSCR) of Asphalt Binders using a Dynamic Shear Rheometer, ASTM Standard D7045, 2010.

[15] Performance-Graded Asphalt Binder Using Multiple Stress Creep Recovery (MSCR) Test, AASHTO MP19, 2010. 